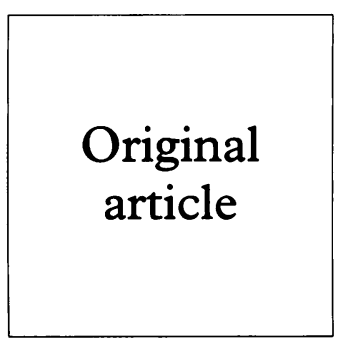

\title{
Genital Chlamydia trachomatis (serotypes D-K) infection in Jamaican commercial street sex workers
}

\author{
G Dowe, S D King, A R Brathwaite, Z Wynter, R Chout
}

Objectives: To determine the prevalence of genital Chlamydia trachomatis infections in commercial street sex workers (CSSW) in Jamaica.

Methods: The prevalence of $C$ trachomatis infection was determined in 129 Jamaican CSSW using the direct fluorescent antibody (DFA) method and the isolation techniques which utilise fluorescent and iodine staining of endocervical cytobrush specimens cultured in McCoy cells. The seroprevalence of $C$ trachomatis in the CSSW was also compared with that in blood donors ( $\mathrm{n}=$ 435), using the microimmunofluorescence (MIF) test.

Results: The DFA detected $C$ trachomatis in $16 \%(21 / 129)$ of the specimens. The prevalence as determined by the iodine and fluorescein stained cultures was $24 \%(31 / 129)$ and $25 \%(33 / 129)$ respectively. The overall prevalence of current chlamydial infection detected by the isolation techniques used was $25 \%$ (33/129). As determined by the MIF test, a statistically significantly higher seroprevalence rate of $C$ trachomatis $(95 \%, 61 / 64)$ was found in CSSW compared with blood donors $\left(53 \%, 229 / 435\right.$; OR $\left.22 \cdot 6 ; \chi^{2}=49 \cdot 8 ; p<0 \cdot 001\right)$. The prevalence of current infection in CSSW as indicated by the isolation of $C$ trachomatis was not influenced by history of previous pelvic inflammatory disease (PID), sexually transmitted disease, or condom use. $N$ gonorrhoeae (9\%) and Candida albicans (7\%) were found in comparatively low frequencies, while Trichomonas vaginalis $(0 \%)$ was not found in specimens from the CSSW.

Conclusions: A high seroprevalence rate and a high rate of current infection with $C$ trachomatis occur in Jamaican CSSW. In order to control the spread and prevent the severe clinical complications and sequelae of $C$ trachomatis infection, the diagnosis and treatment in such high risk groups such as CSSW should be optimised.

(Genitourin Med 1997;73:362-364)

Keywords: sex workers; chlamydial infection; Jamaica

\section{Introduction}

Genital infection caused by Chlamydia trachomatis serotypes $\mathrm{D}-\mathrm{K}$ is currently the most common bacterial sexually transmitted disease (STD) reported in developed countries. ${ }^{12} \mathrm{~A}$ high prevalence of chlamydial infections also occurs in developing countries including the Caribbean. ${ }^{12}$

In women, infections caused by $C$ trachomatis may cause severe acute complications such as pelvic inflammatory disease (PID) leading to serious sequelae including premature rupture of membranes, ectopic pregnancy, and infertility. ${ }^{2}$

King et $a l^{3}$ reported a high prevalence of genital $C$ trachomatis infection in STD, gynaecological, and family planning clinic clients in Jamaica. ${ }^{3}$ Prostitutes constitute a high risk group for $C$ trachomatis infection. ${ }^{14}$ This study was undertaken to determine the prevalence of $C$ trachomatis infection in Jamaican commercial street sex workers (CSSW).

Patients, methods, and materials One hundred and twenty nine female CSSW (mean age 25 years, range 17-52) from Kingston were enrolled in the study. For recruiting this group, consecutive CSSW were approached, in the street, by one of our collaborators and invited to participate in the study. Four hundred and thirty five blood donors (300 men, 135 women; mean age 30 years, range 19-59) were randomly selected at the central blood bank in Kingston. A questionnaire pertaining to age, residence, sexual behaviour, urogenital problems, contraceptive use, and previous sexually transmitted disease was administered to each CSSW by one of the authors (GD).

During a full gynaecological examination, endocervical specimens (Dacron swab and cytobrush samples) were obtained from each CSSW. The external genitalia were examined for lesions and a speculum used to detect any cervical lesion or inflammation. The exocervix was cleaned before the swab was inserted into the endocervix. A wet preparation of the endocervical swab was examined microscopically for Trichomonas vaginalis. ${ }^{5}$ Gram stain and culture procedures for the isolation of $N$ gonorrhoeae and Candida albicans were also performed on the endocervical swab. ${ }^{5}$ The cytobrush samples were examined for $C$ trachomatis by the direct fluorescent antibody (DFA) method using Micro Trak kits (Syva Palo Alto, CA, USA). ${ }^{6}$ The same sample was placed in duplicate McCoy cell shell vial cul- 
tures as previously described. ${ }^{6}$ Cultures were harvested and stained with iodine and fluorescent monoclonal antibodies to $C$ trachomatis (Syva Micro Trak) respectively. ${ }^{6}$ In the DFA test a smear containing 10 or more elementary bodies was considered positive. ${ }^{6}$ Shell vial cultures were taken as positive if characteristic inclusion bodies were seen by immunofluorescence or iodine staining. ${ }^{6}$

Clotted blood samples $(5 \mathrm{ml})$ were obtained from 64 of the CSSW. The sera were tested for antichlamydial antibodies by a commercially available microimmunofluorescence (MIF) test which detected IgG antibodies (Bio Mereiux, France).

Data were compared by $\chi^{2}$ analyses and Wilcoxon's test.

\section{Results}

The results of the chlamydia isolation techniques are summarised in table 1 . Using the DFA technique $16 \%(21 / 129)$ of the endocervical cytobrush specimens were positive for $C$ trachomatis. When the specimens were cultured $C$ trachomatis was detected by iodine staining in $24 \%(31 / 129)$ and by fluorescent monoclonal antibody in $25 \%$ (33/129). Compared with culture, the specificity of the DFA was $100 \%$, but the sensitivity was less $(68 \%)$. The fluorescent monoclonal antibody staining method detected all the positives identified by iodine staining. The overall rate of isolation of $C$ trachomatis by DFA and culture techniques was $25 \cdot 0 \%$ (33/129).

The MIF test for chlamydial antibodies was performed on the 64 serum samples from the CSSW and the 435 blood donors. The seropositivity rate in CSSW (95\%, 61/64) was statistically significantly higher than that observed in blood donors (53\%, 229/435; OR $\left.22.6 ; \chi^{2}=49.8, p<0.001\right)$. No correlation was observed between MIF reciprocal titres and the presence of $C$ trachomatis isolates.

As shown in table 2, the most frequent clinical manifestation of $C$ trachomatis infection in the CSSW was vaginal discharge. This

Table 1 Prevalence of Chlamydia trachomatis in 129 famaican commercial street sex workers using different methods

\begin{tabular}{ll}
\hline Method & No positive (\%) \\
\hline Direct immunofluorescence & $21(16)$ \\
$\begin{array}{l}\text { Tissue culture iodine stained } \\
\text { Tissue culture fluorescent monoclonal }\end{array}$ & $31(24)$ \\
antibody stained & $33(25)$ \\
\hline
\end{tabular}

Table 2 Clinical manifestations of Chlamydia trachomatis infection in famaican commercial street sex workers $(n=129)$

\begin{tabular}{lll}
\hline Manifestation & $\begin{array}{l}\text { Chlamydia } \\
\text { isolate } \\
\text { negative } \\
(n=96)\end{array}$ & $\begin{array}{l}\text { Chlamydia } \\
\text { isolate } \\
\text { positive } \\
(n=33)\end{array}$ \\
\hline Vaginal discharge & $74(77)$ & $25(76)$ \\
Vaginal pruritus & $20(21)$ & $8(24)$ \\
Genital sores/ulcers & $11(11)$ & $5(15)$ \\
Lower abdominal pain & $31(32)$ & $14(42)$ \\
Abnormal cervix & $48(50 \cdot 0)$ & $15(45)$ \\
\hline${ }^{\star}$ Includes friability, erosions, oedema, erythema, and ulcer.
\end{tabular}

occurred in similar frequencies in both $C$ trachomatis positive $(76 \%, 25 / 33)$ and negative (77\%; 74/96) CSSW.

$C$ trachomatis positivity in CSSW did not correlate significantly with previous history of PID (55\% versus $44 \%$ ), STD (25\% versus $27 \%$ ), or the use of condoms (23\% versus $37 \%)$.

Neisseria gonorrhoeae was isolated from $9 \%$ $(11 / 129)$ of the endocervical swabs taken from the CSSW, Candida albicans from $7 \%(9 / 129)$, while Trichomonas vaginalis was not seen. Of the $11 \mathrm{CSSW}$ who had $N$ gonorrhoeae only one (9\%) had concomitant infection with $C$ trachomatis.

\section{Discussion}

The prevalence of current $C$ trachomatis infection in Jamaican CSSW as indicated by the isolation techniques used in this study was $25 \%$. The DFA test used in this study was adequately specific, but was less sensitive than culture for the isolation $C$ trachomatis. Though not widely available, polymerase chain reaction and ligase chain reaction currently are the most sensitive techniques for detecting cervical chlamydia infection. The percentage of Jamaican CSSW with current $C$ trachomatis infection observed in this study was comparable with that reported in prostitutes from developed as well as developing countries. ${ }^{47}$

Compared with the general Jamaican population, a significantly higher percentage of CSSW had current or past infection with $C$ trachomatis as shown by the MIF test results. High seroprevalence rates of $C$ trachomatis have been reported in prostitutes in other developing countries. ${ }^{4}$ However, MIF tests for IgG as well as IgM antichlamydial antibodies are of limited value as indicators of current infection. $^{8}$

In this study infection with $C$ trachomatis was not influenced significantly by history of previous STD, PID, or condom use. Yet it is well documented that the use of non-barrier methods of contraception such as oral contraceptives, is a risk factor for the acquisition of $C$ trachomatis infection. ${ }^{9}$ Vaginal discharge was the most frequently occurring manifestation of $C$ trachomatis infection experienced by the CSSW. Although one $C$ trachomatis positive CSSW had concomitant $N$ gonorrhoeae infection, other causes were not entirely ruled out as causes of vaginal discharge in this series of CSSW.

Notably, the other STD pathogens sought in this study were less prevalent than chlamydia in Jamaican CSSW. It was of interest that the prevalence of gonococcal infection in Jamaican CSSW was lower than the $21 \%$ reported recently in Jamaican women of child bearing age. ${ }^{10}$ Increasing trends in the prevalence of both trichomonal and candidal infections have also been reported in Jamaican women in this age group. ${ }^{10}$ The low prevalence of $N$ gonorrhoeae and low occurrence of concomitant infection with $C$ trachomatis indicate that chlamydial co-treatment of gonorrhoea by itself is not effective as a control strategy for 
chlamydia in this population. This study confirms the predominance of $C$ trachomatis among the bacterial causes of STD and the tendency for a high prevalence of this infection to occur in high risk groups.

A high seroprevalence rate and a high rate of current infection with $C$ trachomatis occur in Jamaican CSSW. Potentially this group is an important source of $C$ trachomatis in the Jamaican population. In order to control the spread and prevent the severe clinical complications and sequelae of $C$ trachomatis infection, diagnosis and treatment in high risk groups such as CSSW should be optimised.

1 Venegas VS, Villafranca $P$, Madrid JP, Cosenza $H$ Bygdeman S. Gonorrhoea and urogenital chlamydia infection in female prostitutes in Tegucigalpa, Honduras. Int $\mathcal{F}$ STD AIDS 1991;2:195-9.
2 Ronsmans C, Bulut A, Yolsal N, Agacfidan A, Filippi V. Clinical algorithms for the screening of Chlamydia tra chomatis in Turkish women. Genitourin Med 1996;72 182-6.

3 King SD, Dowe G, Wynter HH, Brathwaite AR. Chlamydial infections in selected populations in Jamaica. WI Med f 1992;42:136-8.

4 Reeves WC, Quiroz E. Prevalence of sexually transmitted diseases in high risk women in the Republic of Panama. Sex Transm Dis 1987;14:69-74.

5 Morse SA, Sarafian SK Sexually transmitted diseases. Manual of clinical microbiology. 5th ed. Washington DC: American Society for Microbiology, 1991:863.

6 Barnes RC. Laboratory diagnosis of human chlamydial infections. Clin Microbiol Rev 1989;2:119-36.

7 Stary A, Kopp W, Gebhart W, Soltz-Szots J. Culture versus direct specimen test: comparative study of infections with Chlamydia trachomatis in Viennese prostitutes. Genitourin Med 1985;61:258-60.

8 Mardh PA, Paavonen J, Puolakkainen M. Chlamydia. New York and London: Plenum Press, 1989.

9 Fraser J, Rettig JD, Kaplan DW. Prevalence of cervical Chlamydia trachomatis and Neisseria gonorhoeae in female adolescents. Pediatrics 1983;71:333-6.

10 The Epidemiology Unit, Ministry of Health, Jamaica. Sexually Transmitted Disease (STD) Control Programme Annual Report. Kingston: Ministry of Health, 1993. 